\title{
APPLICATION OF SOLID STATE NUCLEAR TRACK DETECTORS FOR PERSONNEL MONITORING AROUND HIGH ENERGY ACCELERATORS
}

\author{
J. Dutrannois and J.W.N. Tuyn \\ CERN, Geneva \\ Switzerland
}

A B S T R A C T

In the framework of the design of a better system for personnel neutron monitoring, an extensive systematic study has been carried out to evaluate the feasibility of different nuclear track detector-radiator combinations.

This paper is concentrated on the use of LRIl5 cellulose nitrate and Makrofol polycarbonate foil with different etching and counting techniques. For LRI15, classical etching was applied and automatic track counting and analysis were performed with a Quantimet. For the Makrofol, in addition to the standard spark counting method, electrochemical etching was used. Because of the wide neutron energy spectrum to be covered it was necessary to use a selection of radiators. It appears that an empirical formula for the neutron dose can be derived from the readings of the track density under the chosen radiators consisting of plastic, boron-loaded material, and bismuth. A comparison of the results obtained with this method and the dose recorded by standard radiation survey instrumentation has been performed.

\section{INTRODUCTION}

In the radiation environment of high energy proton accelerators the problem of personnel neutron monitoring is impossible to solve satisfactorily by the use of either nuclear emulsions or simple albedo dosimeters because of the strong variation of the neutron spectrum in time and space, the more so since the neutron spectrum covers an extremely wide energy range. An approach to solve this problem at CERN by the use of solid state nuclear track detectors in conjunction with different radiators has been reported elsewhere $(1,2)$ whereby for example ${ }^{6}$ LiF thermoluminescence detectors (TLD) were used as TL detectors as well as radiators for cellulose nitrate. The present paper describes the results of a further study of the feasibility of different nuclear track detector-radiator combinations for personnel neutron monitoring. Basically two different detector 
materials were used: Makrofol polycarbonate and LRII5 cellulose nitrate foil with different etching and counting techniques. The wide neutron energy spectra to be covered necessitated the use of at least two different radiators for an acceptable neutron dose estimation, as will be shown by results of field tests obtained at different realistic positions around the CERN high energy proton accelerators compared with the standard radiation survey instrumentation used at CERN. Results obtained with fissile radiators are included for comparison only, since it is felt that such radiators owing to their radiotoxicity are not recommended for personnel dosimetry but should be reserved for example for neutron fluence measurements.

\section{EXPERIMENTAL PROCEDURES}

The series of investigations were concentrated on two different detector materials:

- LRII5 foil consisting of an $8 \mu \mathrm{m}$ thick layer of cellulose nitrate incorporating a red dye attached to a transparent polyester backing.

- Makrofol E polycarbonate foil in two different thicknesses: $10 \mu \mathrm{m}$ for use with the spark counting technique developed by Cross and Tommasino (3) and $500 \mu \mathrm{m}$ for use with the technique of electrochemical etching developed by Tommasino (4) and described in detail in another paper at this conference (5).

The LRII5 foil was used with three different radiators: an $8 \mu \mathrm{m}$ layer of natural boron on glass backing for detection of low energy neutrons through the ${ }^{10} \mathrm{~B}(\mathrm{n}, \alpha)^{7} \mathrm{Li}$ reaction, polythene for detection of fast neutrons mainly through $C$ recoils arising from neutron interactions in radiator as well as detector foil, and finally $\mathrm{Bi}$ for additional contributions of reaction products from spallation and fission reactions induced by high energy particles. The LRII5 foils were etched for 90 minutes in a $2.5 \mathrm{~N} \mathrm{NaOH}$ solution at $60^{\circ} \mathrm{C}$, while the induced tracks which appear under a microscope as light spots on a dark ground were counted by using a Quantimet, an image analysing computer, which not only counts the track density but evaluates the hole size distribution as well. This is shown in Figs. 1 and 2, where the size distribution as a function of hole 
diameter is given for the areas under the different radiators. As can be seen, the reaction products of the ${ }^{10} B(n, \alpha)^{7} \mathrm{Li}$ reaction cause a significantly different distribution compared to the two other radiators, where holes caused by heavier particles are dominating. For routine use all holes exceeding a threshold diameter of $7.4 \mu \mathrm{m}$ are counted to discrimate against unwanted background. A total surface of $0.2 \mathrm{~cm}^{2}$ is scanned routinely.

The spark counting technique was used for the $10 \mu \mathrm{m}$ Makrofol foils in contact either with Makrofol or with Th and Bi foils as fissile radiators. In the first case neutron induced $\mathrm{C}$ and 0 recoils from the radiator or the detector foil itself are detected by etching the foils for 72 hours in a $6.25 \mathrm{~N} \mathrm{KOH}$ solution at room temperature, after which only $2 \mu \mathrm{m}$ of the foil is left, necessary because of the short range of the recoils. For fissile radiators 22 hours of etching is sufficient. The tracks were after etching enlarged at $900 \mathrm{~V}$ and counted at $550 \mathrm{~V}$. Typical recoil induced sparks versus voltage graphs are shown in Fig. 3 .

Finally the electrochemical etching technique with $500 \mu \mathrm{m}$ thick Makrofol foil was used according to the optimal conditions mentioned elsewhere (5): $2.8 \mathrm{kV}$ at $3 \mathrm{kHz}$ in a $1: 1$ mixture of $25 \% \mathrm{KOH}$ and $\mathrm{C}_{2} \mathrm{H}_{5} \mathrm{OH}$ at room temperature for 3 hours.

\section{RESULTS AND DISCUSSION}

Irradiations of foils were performed at different locations outside the shielding of the CERN $28 \mathrm{GeV}$ proton synchrotron at which the neutron spectrum was known to vary strongly. The results obtained were compared with the dose equivalent obtained from the standard CERN survey instrumentation consisting of a Rem Ion Chamber (RIC) for neutrons up to $\sim 20 \mathrm{MeV}$ and ${ }^{1 l_{C}}$ activation detectors for the detection of hadrons above $20 \mathrm{MeV}$. The RIC moderator was used as a phantom as well. The ratio of the dose equivalent measured by these two devices is a sensitive neutron spectrum index. In a previous study an empirical relationship for the neutron dose equivalent derived from the hole densities in LRIl5 behind a ${ }^{6}$ LiF teflon thermoluminescent detector disc and a polythene cover was found to give an estimation within $\sim 20 \%$ of the neutron dose (6). For practical 
reasons the new CERN proposed personnel monitor uses the ${ }^{6}$ LiF TL detector only as a neutron indicator and separately boron as a radiator. Taking into account the higher hole density under the boron radiator the revised relationship becomes:

H $($ mrem $)=5.2\left(\frac{\text { holes } / \mathrm{cm}^{2} \text { under B }}{600}+\right.$ holes $/ \mathrm{cm}^{2}$ under polythene $)$

The hole density under the $B i$ radiator has not been included in eq. (1) in spite of the higher hole density compared to polythene because of its higher background due to the presence of natural alpha emitters in $\mathrm{Bi}$.

The results obtained for three locations with the detectors attached to front (F) and back (B) of the moderator in strongly varying neutron spectra as can be seen from the $\mathrm{H}_{\mathrm{HEP}} / \mathrm{H}_{\text {neutron }}$ ratio are presented in table I. Relation (I) approximates the dose for the hard spectrim with the accuracy as found in the past. For the medium and soft spectra an overestimation is made, partly owing to the statistical error in the number of holes under the polythene, partly to the high and locally strongly varying contribution of low energy neutrons.

The neutron sensitivity of Makrofol after electrochemical etching is somewhat lower for fast neutrons, while its sensitivity for low energy neutrons underneath the boron radiator is higher than LRII5. Therefore, already at low doses, overlapping of tracks is a problem since the tracks are enlarged considerably by electrochemical etching, as can be seen from the size distribution in Fig. 4, where the distribution for foils with and without overlapping tracks are shown. The maximum in the distribution is found around a diameter of $0.5 \mathrm{~mm}$, which is considerably larger than for LRl15 (see Figs. 1 and 2) with $0.02 \mathrm{~mm}$. Therefore scanning of Makrofol can be done at a lower magnification than LRI15. Background elimination in LRII5 is however simpler with the Quantimet than in Makrofol. In the latter, background is reduced by electrochemical and conventional etching before irradiation (5) and the distinction between background and newly induced tracks still requires some human judgement. For both methods background subtraction is the main limitation. We found that LRII5 background varies from one 
badge to the next and even storage at different temperatures may considerably influence the background. For the LRII5 samples used in this study a background of $56 \pm 24 \mathrm{holes} / \mathrm{cm}^{2}$ was found. The pre-etched background of Makrofol varied between 50 and 120 tracks $/ \mathrm{cm}^{2}$ for the foils used. The background to be subtracted after pre-etching is much lower, $\sim 15$ tracks $/ \mathrm{cm}^{2}$. The lower background combined with the lower magnification and consequently larger surface scanned is reflected in the higher statistical accuracy for Makrofol compared to LRII5 in Table I.

The use of the spark counting method was tested under different conditions with PuBe neutrons, neutrons produced by $600 \mathrm{MeV}$ protons on a Be target, and outside the shielding of the PS West Hall. The results are given in table II in comparison with some measurements with $500 \mu \mathrm{m}$ thick Makrofol. A few conclusions can be drawn: the directional dependence with sparks due to recoil particles is much stronger than with the electrochemically etched Makrofol for PuBe neutrons, and the neutron energy dependence is strong for the spark counting method regardless of whether Th or Makrofol is used as radiator. It has been shown in the past that the $B i / T h$ ratio could be used as a spectrum index to estimate the sparks/rem for Th covered foils (2). However, the sensitivity of $\mathrm{Bi}$ is low. In spite of its stronger energy and directional dependence, Makrofol without fissile radiators should be preferred for personnel neutron dosimetry to avoid the spread of radioactive material. Unfortunately the fact that for recoil-induced sparks the foils have to be etched down to a thickness of $2 \mu \mathrm{m}$, close to electrical breakdown, makes the method extremely sensitive to variations in etching conditions or foil thickness. The sensitivity of the method is sufficient for personnel neutron monitoring around accelerators since a surface of $20 \mathrm{~cm}^{2}$ is easily sparkable and would produce $\sim 800$ sparks/rem in addition to 10 sparks of background. Another application was found in measuring the depth distribution of high LET particles in a polythene absorber in front of the $600 \mathrm{MeV}$ beam. The preliminary result is shown in Fig. 5 compared with the depth-dose distribution measured with a tissue-equivalent ionization chamber. The interpretation of this distribution might be difficult because of its possible dependence on etching conditions as shown by Becker et al. (7). 


\section{CONCLUSIONS}

The results obtained with the three different techniques described in this paper lead to the following conclusions.

The spark counting method for recoils has sufficient sensitivity and an attractive lower detection limit for personnel neutron monitoring around high energy proton accelerators because of its low background. The strong energy and directional dependence combined with the possible errors in the evaluation procedure are reasons to prefer the use of LRII5 or Marrofol electrochemically etched. The final choice for use at CERN to replace the nuclear emulsion for personnel neutron monitoring will strongly depend upon the way in which the background of the foils can be further reduced by better preparation techniques for LRII5 or an improved pre-etching procedure for Makrofol. The routine large scale electrochemical etching of Makrofol may create additional practical problems.

\section{ACKNOWLEDGEMENTS}

The contributions of G.M. Hassib, S. Hertzman and M. Hofert to this study is gratefully acknowledged.

\section{REFERENCES}

1. B.J. Tymons, J.W.N. Tuyn and J. Baarli, Proc. Symp. Neutron Monitoring for Protection Purposes, IAEA, Vienna, 1972, Vol. II, p. 63 .

2. B.J. Tymons, J. Dutrannois and J.W.N. Tuyn, Working Party on Space Biophysics, Geneva, 1973, CERN Report DI/HP/168.

3. N.G. Cross and L. Tommasino, Proc. Int. Topical Conf. on Nuclear Track Registration in Insulating Solids and Applications, 1969, Vol. 1, p. 73 .

4. L. Tommasino, CNEN Report RT/PROT/71)I (1970).

5. G.M. Hassib, J.W.N. Tuyn and J. Dutrannois, On the electrochemical etching of neutron induced tracks in plastics and its application to personnel neutron dosimetry, this conference.

6. B.J. Tymons and J.W.N. Tuyn, Personnel neutron dosimetry by means of cellulose nitrate film combined with LiF as both radiator and TLD, Health Physics (in press).

7. K. Becker and M. Abd-el-Raseh, ORNL-TM-4460 (1974). 


\section{FIGURE CAPTIONS}

Fig. 1. Track diameter distribution in LRII5 behind various radiators for a "medium" neutron spectrum.

Fig. 2. Track diameter distribution in LRll5 behind various radiators for a "hard" neutron spectrum.

Fig. 3. Spark counts as a function of sparking voltage after 72 hours etching.

Fig. 4. Track diametex distribution in Makrofol electrochemically etched behind a boron radiator.

Fig. 5. Depth-dose distribution in a $600 \mathrm{MeV}$ neutron beam compared with a recoil spark distribution. 
Table I

Comparison between LRII5 and Makrofol (electrochemically etched) in different neutron spectra

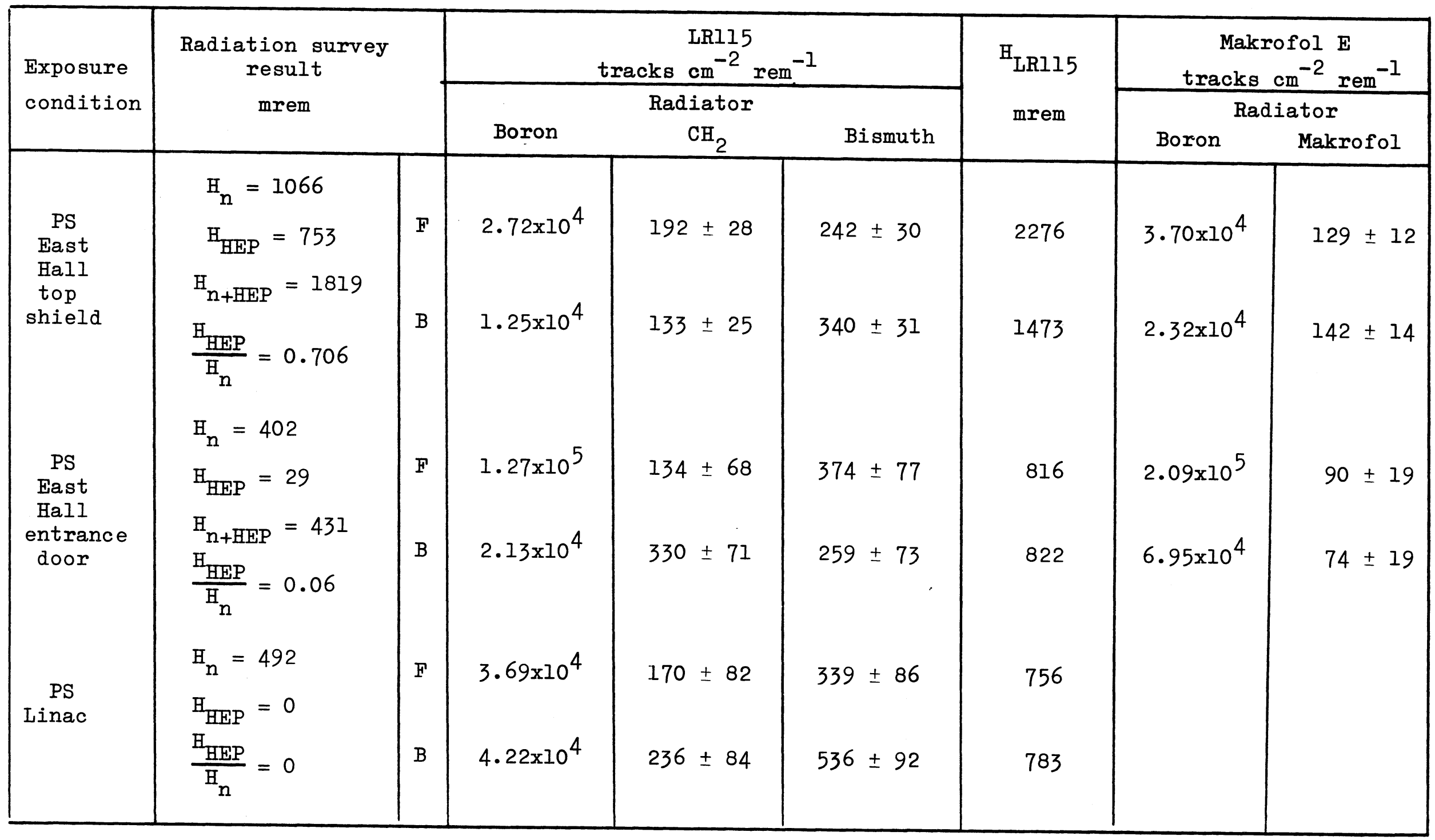




\section{Table II}

Comparison of neutron sensitivity and directional dependence of various detectors

\begin{tabular}{|c|c|c|c|c|}
\hline \multirow[t]{3}{*}{ Exposure condition } & \multirow{3}{*}{$\begin{array}{c}\text { Makrofol } \\
\text { (electrochemically etched) } \\
\text { tracks } \mathrm{cm}^{-2} \mathrm{rem}^{-1}\end{array}$} & \multicolumn{3}{|c|}{$\begin{array}{c}\text { Makrofol (spark counting) } \\
\text { sparks } \mathrm{cm}^{-2} \mathrm{rem}^{-1}\end{array}$} \\
\hline & & & Radiator & \\
\hline & & Makrofol & Thorium & Bismuth \\
\hline \multicolumn{5}{|l|}{ PuBe neutrons: } \\
\hline Normal incidence & 50 & 4.2 & 18 & \\
\hline $45^{\circ}$ incidence & 30 & 2.1 & & \\
\hline Parallel incidence & 15 & 0.42 & & \\
\hline $\begin{array}{l}\text { PS West Hall } \\
\frac{\mathrm{H}_{\mathrm{HEP}}}{\mathrm{H}_{\mathrm{n}}}=0.56\end{array}$ & & 43 & 73 & 5.5 \\
\hline SC $\underset{\text { beam }}{600 \mathrm{MeV}}$ neutron & & 10 & 109 & 11 \\
\hline
\end{tabular}




\section{hard spectrum}

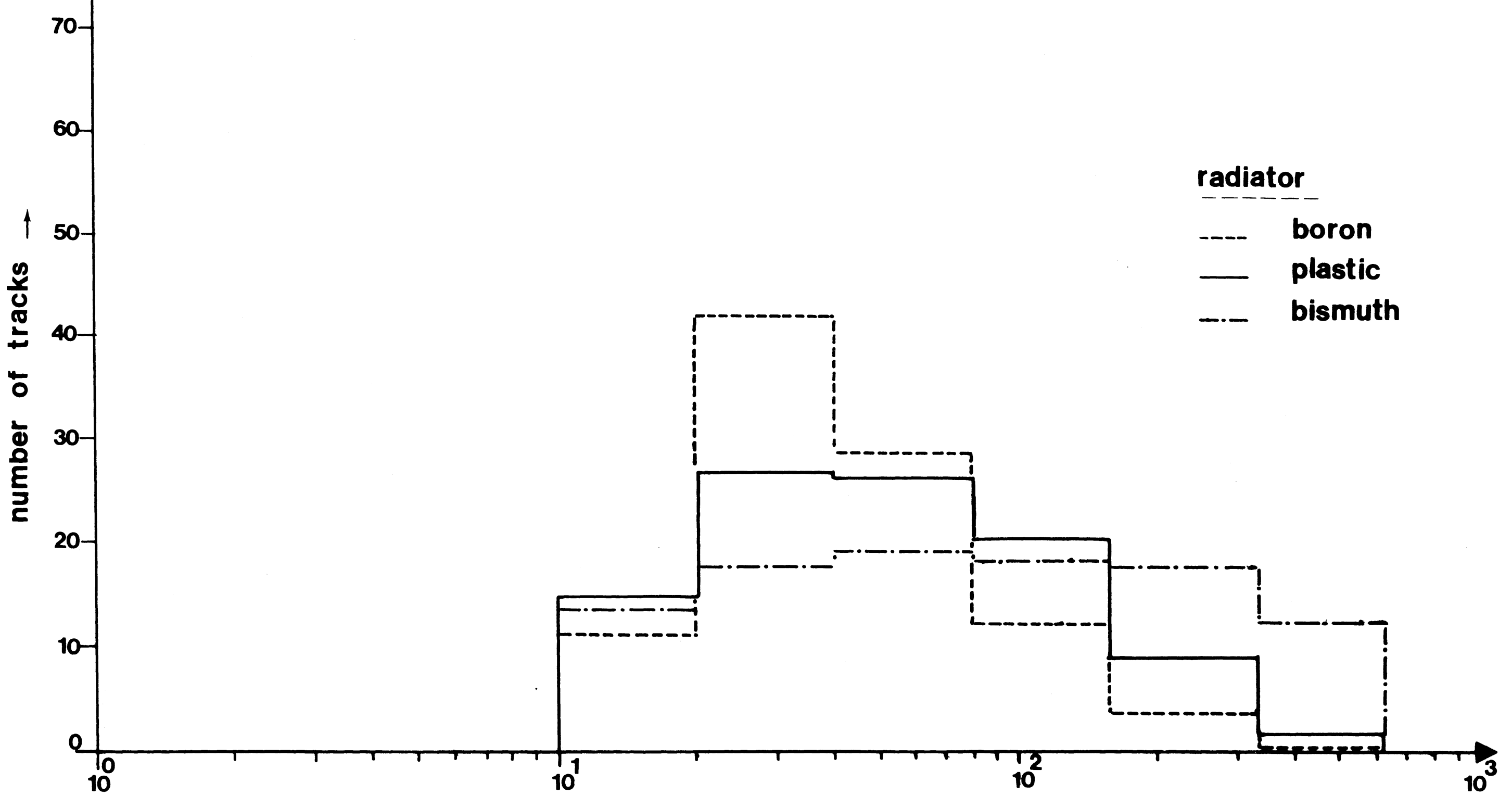

track diameter in number of image points $\rightarrow$ 


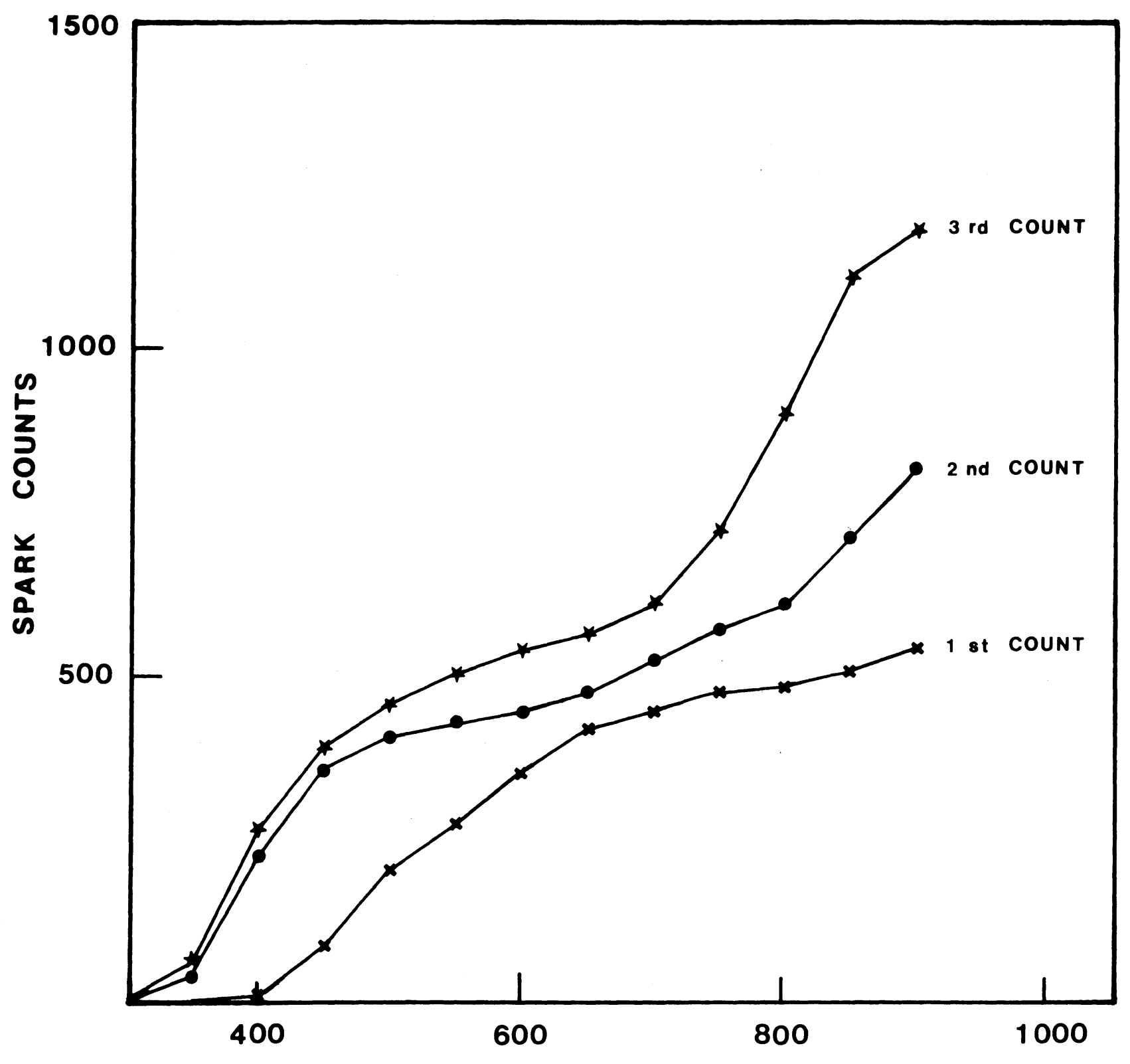

SPARKING VOLTAGE (V)

F IG . 3 


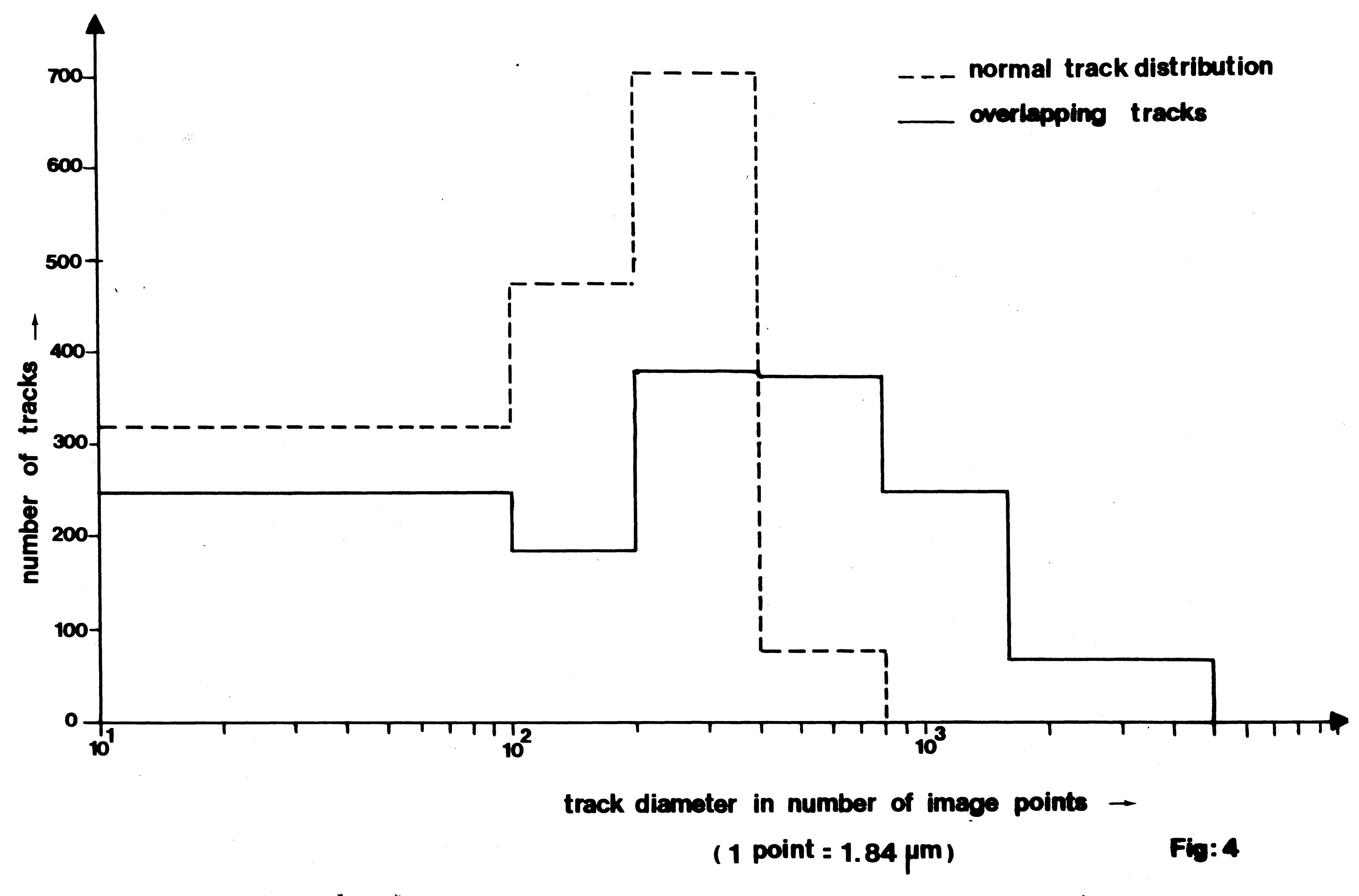




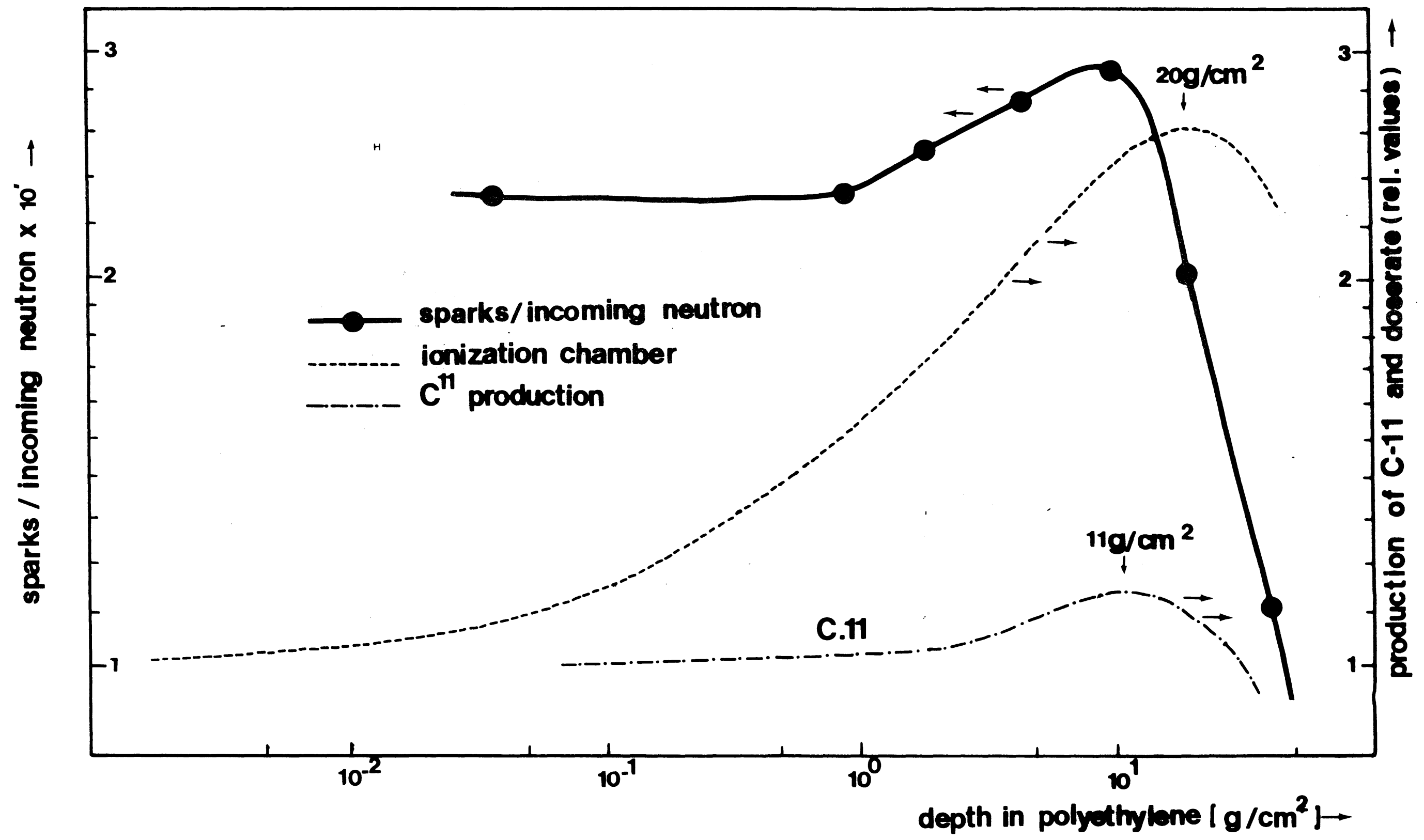

Fig:5 